University of Wollongong

Research Online

Faculty of Engineering - Papers (Archive)

Faculty of Engineering and Information

Sciences

2003

\title{
A non-destructive, non-contact, quality test of critical current for Ag-BiSCCO tape
}

A. Gandini

University of Houston, USA

R. Weinstein

University of Houston, USA

D. Parks

University of Houston, USA

R. Sawh

University of Houston, USA

S. X. Dou

University of Wollongong, shi@uow.edu.au

Follow this and additional works at: https://ro.uow.edu.au/engpapers

Part of the Engineering Commons

https://ro.uow.edu.au/engpapers/53

\section{Recommended Citation}

Gandini, A.; Weinstein, R.; Parks, D.; Sawh, R.; and Dou, S. X.: A non-destructive, non-contact, quality test of critical current for Ag-BiSCCO tape 2003.

https://ro.uow.edu.au/engpapers/53

Research Online is the open access institutional repository for the University of Wollongong. For further information contact the UOW Library: research-pubs@uow.edu.au 


\title{
A Non-Destructive, Non-Contact, Quality Test of Critical Current for Ag-BiSCCO Tape
}

\author{
Alberto Gandini, Roy Weinstein, Drew Parks, Ravi-Persad Sawh, and Shi Xue Dou
}

\begin{abstract}
An accurate noncontact method of characterizing Ag-BiSCCO tape is reported, using the trapped field, $B_{t}$, of a field-cooled tape. The dimensions of the tape make it possible to calculate in closed form a very simple expression, relating trapped field to transport current. Short lengths of tape, $4(\mathrm{~cm})$, are used to experimentally confirm the model. The model applies to any length of tape for which length is greater than 5 times the tape width. A schematic of a proposed test apparatus is presented which should permit on-line testing for magnitude of the current, and for cracks, while the tape is in motion during production.
\end{abstract}

Index Terms-BiSCCO tape, critical current density, high temperature superconductors, trapped magnetic field.

\section{INTRODUCTION}

$\mathbf{L}$ ONG tapes of Ag/Bi-2223, made by powder-in-tube processes, are now being produced commercially [1] as well as by university research groups [2]. It is useful prior to completion of the final product to discover any faults which reduce the current carrying capacity of the tape. This information may then be used as part of the continuous process. Contact measurements are undesirable because generally they require the tape production to be stopped. In addition, the tape is subject to fracture by the contact forces.

The approach we use to determine the transport current is to measure the trapped field, $B_{t}$, by a Hall probe. Motivation for this approach is based upon its success in characterizing trapped field magnets (TFM's) [3]-[6]. For example, TFM's of Y-123 can be evaluated for faults, cracks, multi-grain structure, etc. Fig. 1(a) is a graph of $B_{t}(x, y)$ on the surface of a single grain TFM, a cylinder of dimensions $2.0 \mathrm{~cm}$ diameter, and $0.8 \mathrm{~cm}$ height. Fig. 1(b) is a similar sample, which has been cracked under magnetic pressure [7]. We note that at the time of the measurement $B_{t}$ the applied field was removed.

Hall probe measurements have been previously used also to detect inhomogeneities in the current pattern in mono-filamentary and multi-filamentary Ag/BiSCCO tapes [8]-[10].

Manuscript received August 5, 2002. This work was supported in part by the U.S. Army Research Office, the State of Texas via the Texas Center for Superconductivity and Advance Material (TCSAM), the Welch Foundation, and the Australian Research Council.

A. Gandini, R. Weinstein, D. Parks, and R.-P. Sawh are with the Department of Physics and Texas Center for Superconductivity and Advance Material (TCSAM), University of Houston, Houston, TX 77204-5005 USA (e-mail: Agandini@UH.EDU).

S. X. Dou is with the Institute of Superconducting and Electronic Materials, University of Wollongong, NSW, Australia.

Digital Object Identifier 10.1109/TASC.2003.812305

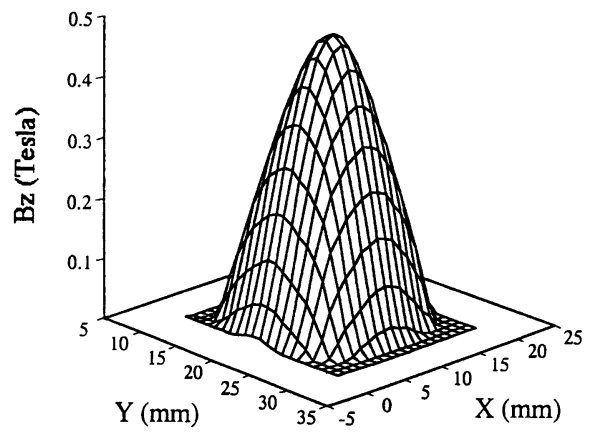

(a)

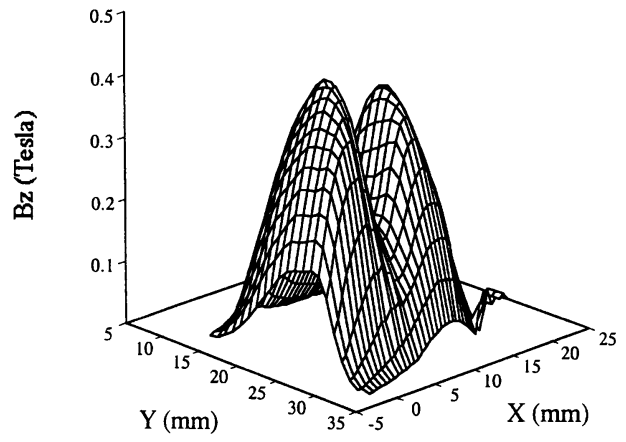

(b)

Fig. 1. (a) Trapped field observed on YBCO samples $2 \mathrm{~cm}$ diameter and $0.8 \mathrm{~cm}$ tall. Result for a single grain. (b) Trapped field observed on YBCO samples $2 \mathrm{~cm}$ diameter and $0.8 \mathrm{~cm}$ tall. Result for a grain cracked under magnetic pressure.

\section{THEORETICAL DESCRIPTION}

To estimate the critical current, $I_{c}$, by measuring $B_{t}$ we apply the Bean model [11], i.e., we assume an uniform current flow through the sample. This current sustains the trapped field, $B_{t}$.

We represent the $\mathrm{Ag} / \mathrm{Bi}-2223$ tape, of width $W$, as two parallel tapes, each of width $W / 2$. We assume the width of the BiSCCO equals the width of the tape. When supporting trapped field perpendicular to the plane of the tape ( $y$ direction) these two tapes each carry current $I_{c} / 2$, in opposite directions. We approximate the long dimension of the tape, $L$, as infinite and the thickness, $t$, as infinitesimal. We denote by $h$ the height of the Hall probe above the tape, and by $\delta$ the horizontal displacement of the probe from the center line of the tape (the $x$ axis). We approximate the Hall probe as a point.

In order to derive a relationship between $B_{t}$ and $I_{c}$, we consider the tape as a sum of an infinite number of tapes of infinitesimal width, $d x^{\prime}$, each carrying a current $(I / w) d x^{\prime}$. This approximation allows us to readily compute $B_{t}$, as function of $I_{c}$, 


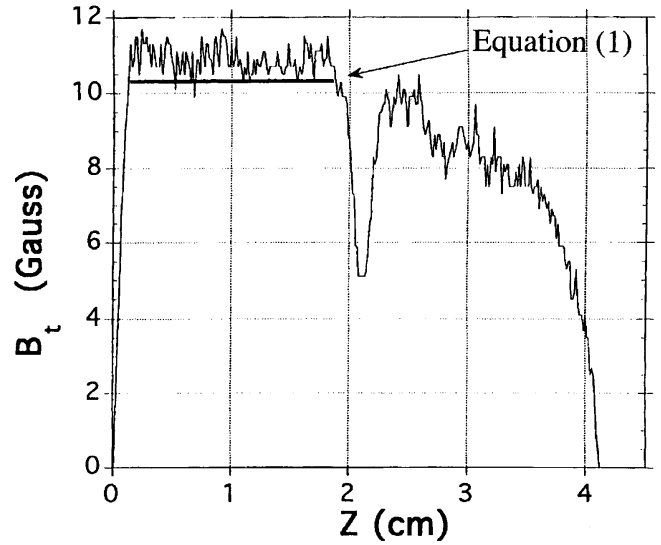

Fig. 2. Trapped field, perpendicular to the surface of an Ag-BiSCCO tape, versus position along length of tape. Uncracked region is between $1.9 \mathrm{~cm}$ and 0 . Continuous line is from (1) for a $I_{c}$ of 29.9 A. Crack is clearly visible at $z \sim 2.2 \mathrm{~cm}$.

by using the Biot-Savart law for each infinitesimal tape and then integrating over $x^{\prime}$. This results in:

$$
B_{t}(x)=\frac{\mu_{0} I}{4 \pi w}\left\{\log \frac{\left[h^{2}+(w / 2+x)^{2}\right]\left[h^{2}+(w / 2-x)^{2}\right]}{\left[h^{2}+x^{2}\right]^{2}}\right\} .
$$

If a more accurate theory is desired, either the two-parameter Kim-Bean model [12] or the simpler two-parameter model [3], [4] can be used. For our purpose here, (1) is sufficiently accurate.

The accuracy of (1) depends on the accuracy of the Bean model, a large value of $R_{L} \equiv L / W$, and the moderately large value of $R_{W} \equiv W / t$. In particular, large values of $R_{L}$ mean that (1) applies to tapes of infinite length.

\section{EXPERIMENTAL}

Several Ag-BiSCCO tapes were scanned longitudinally and transversally by means of a Hall probe attached to an $x-y$ plotter. The samples are $\sim 4 \mathrm{~cm}$ long, $\sim 3.2 \mathrm{~mm}$ wide, and $\sim 1.5 \mathrm{~mm}$ thick. Thus $R_{L}=12.5, R_{W}=2.13$, and (1) should apply well in the limit of infinite length. $I_{c}$ is measured via the four-point technique, using a criterion of $1 \mu \mathrm{V} / \mathrm{cm}$. The current carried at $77 \mathrm{~K}$, in self-field, varies among our samples from 20-32 A.

First, we scanned the trapped field longitudinally along the tape length. The Hall probe was positioned at a distance $h=$ $1 \mathrm{~mm}$ above the center of the tape, such that $x=0$.

The samples were cooled in an applied magnetic field, $B_{A}=$ $15 \mathrm{mT}$ generated by Helmholtz coils. The direction of the applied field was perpendicular to the plane of the tape. Using a Hall probe, the vertical trapped field component was measured at about 150 points along the $z$-axis, parallel to the length of the tape. Fig. 2 shows a plot of $B_{t}$ vs. $z$. First, we consider the region $z<1.9 \mathrm{~cm}$, where the transport critical current measured was $I_{c}=29.9 \mathrm{~A}$. From this, (1) predicts a trapped field of 10.3 Gauss. In this case (1) contained only two parameter $I_{c}$, and $W$. We used $W=3.2 \mathrm{~mm}$. The measured field is 10.9 Gauss, within $6 \%$ of the predicted value, despite the simplified model used.

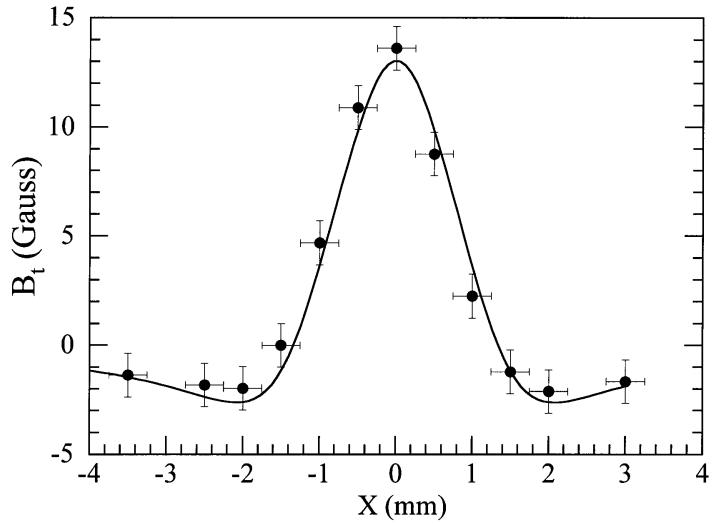

Fig. 3. Trapped field, perpendicular to the surface of an Ag-BiSCCO tape, versus transverse position on the tape $\left(B_{t}\right.$ versus $\left.x\right)$. Continuous line is obtained by the least squared method using (1). The best fitting results in a critical current of $31.9 \mathrm{~A}$, where the transport $I_{c}$ measured by means of the four-point technique yields $I_{c}=31.4 \mathrm{~A}$.

In the region $z<1.9 \mathrm{~cm}$, the measured values of $B_{t}$ vary. This is noise in the Hall probe detector. The value of the standard deviation in this region is $0.041 \mathrm{mT}$. Thus, $\sigma_{B t}=3.8 \% B_{t}$, and the signal to noise for the trapped field is over 26 . For the region of tape with $1.9<z<2.4 \mathrm{~cm}$, Fig. 2 shows the effect of a crack. This shows that, in Ag-BiSCCO tapes, as in Y123 TFM's [5], cracks can be easily seen despite the low trappedfield. The physical crack was very narrow. The structure of the experimental values of $B_{t}$ vs. $z$, near the crack, indicates that spatial resolution in $z$, of the Hall probe, is $\sigma_{z} \sim 2(\mathrm{~mm})$. The space between the Hall probe and the tape, and the size of the Hall probe account for this. A closer and smaller Hall probe will improve the spacial resolution.

Measurements of the trapped field, $B_{t}(x)$ across the width of the tape were also done. Fig. 3 shows the data on trapped field. Data were then fit to (1), with $I_{c}$ as the only free parameter. For this sample, we used $W=2.93 \mathrm{~mm}$ (external tape width), and $h=0.85 \mathrm{~mm}$. By means of least squares method (1) yields $I_{c}=$ $31.9 \mathrm{~A} . I_{c}$ for this tape measured by the four point techniques was $=31.4 \mathrm{~A}$. Thus the predicted and measured currents agree to better than $2 \%$.

\section{DISCUSSION}

We conclude that Ag-BiSCCO tape can be accurately tested for local current, $I_{c}$, and for cracks, in a noncontact, nondestructive way, using trapped field. The agreement between $I_{c}$, measured by the four-point technique, and that measured by a trapped field method is excellent. Both the theoretical model and the experiment should be independent of the length for $B_{t}>0.5 \mathrm{mT}$. In Fig. 4, we show a schematic of a possible test device based upon these considerations. The Ag-BiSCCO tape is fed into a cryostat. This can be as simple as a covered trough, filled with liquid nitrogen. A magnetic field, $B_{A}>B_{t, \max }$ is applied in the region where the tape is cold (i.e., the tape is zero field cooled, ZFC). Here, $B_{t, \max }$ is $B_{t}$ in the critical state. The Bean model indicates that it requires about twice the field to activate to the critical state via $\mathrm{ZFC}$ than via $\mathrm{FC}$. The transducers for this device (a Hall probe, several Hall probes, 


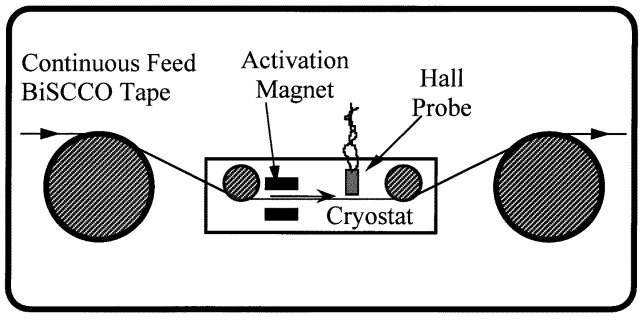

Fig. 4. Schematic of proposed device for an on-line quality characterization of Ag-BiSCCO tape.

or a multi-Hall probe) measure the component of $B_{t}$ perpendicular to the tape surface. One may also apply this approach to measure $B_{t}$ parallel to the surface. The trapped field method for monitoring tape quality is inexpensive, because the only components of any complexity are the Hall probe(s) and their associated electronics. When the tape emerges from the cryostat, it is warmed, and re-spooled, or used as the input for further processing. Thus, the method involves one cooling/warming cycle.

\section{CONCLUSION}

We conclude that measurement of trapped field on Ag-BiSCCO tape can be used, with (1), to calculate absolute transport current to better than $10 \%$. It can be used to measure variations in current larger than $3.8 \%$. It can also locate the position of cracks in the tape to $\sim 2(\mathrm{~mm})$. These resolutions can be further improved by reducing the hall probe area, moving closer to the tape, or averaging over noise.

\section{REFERENCES}

[1] American Superconductor Corp., Westborough, MA.

[2] S. X. Dou and H. K. Liu, Supercond. Sci. and Technol., vol. 6, p. 297, 1993.

[3] J. Liu, I.-G. Chen, R. Weinstein, and J. Xu, Jour. Appl. Phys., vol. 73, p. 6530, 1993.

[4] I.-G. Chen, J. Liu, R. Weinstein, and K. Lau, Jour. Appl. Phys., vol. 72, p. 1013, 1992.

[5] R. Weinstein, Internat. Workshop on Processing and Applications of Large Grain Superconducting (RE)BCO Materials, Cambridge, UK, July 1997. Journal of Material Science and Engineering B, vol. 53, pp. 38-44, 1998.

[6] P. Kottman et al., Superconductor Sci. Technol., vol. 67, p. 7, 1994.

[7] Y. Ren, R. Weinstein, J. Liu, and R.-P. Sawh, Physica C, vol. 251, p. 15, 1995.

[8] P. Kovac, V. Cambel, and P. Bukva, Supercond. Sci. Technol., vol. 12, pp. $465-471,1999$.

[9] A. Tanihata, A. Sakai, M. Matsui, N. Nonaka, and K. Osamura, Supercond. Sci. Technol., vol. 9, pp. 1055-1059, 1996.

[10] M. Polak, M. Majoros, J. Kvitkovic, P. Kottman, P. Kovac, and T. Melisek, Int. Cryogenic Engin. Conf. (ICEC 15), Genoa, Italy, June 7-10, 1994.

[11] C. P. Bean, Phys. Rev. Lett., vol. 8, p. 250, 1962. Revs. Mod. Phys., vol. 36, p. 31, 1964.

[12] Y. B. Kim, C. F. Hempstead, and A. R. Strand, Phys. Rev., vol. 129, p. $528,1963$. 